\title{
Downregulation of microRNA-29c reduces pain after child delivery by activating the oxytocin-GABA pathway
}

\author{
CAIJUAN LI ${ }^{1}$, XIAN WANG ${ }^{1}$, GUANGFEN ZHANG $^{2}$, YAO ZHANG ${ }^{1}$, \\ FAN XIA ${ }^{1}$, SHIQIN XU ${ }^{1}$ and XIAOFENG SHEN ${ }^{1}$ \\ ${ }^{1}$ Department of Anesthesiology, Women's Hospital of Nanjing Medical University, \\ Nanjing Maternity and Child Health Care Hospital; ${ }^{2}$ Department of Anesthesiology, Zhongda Hospital, \\ School of Medicine, Southeast University, Nanjing, Jiangsu 210004, P.R. China
}

Received October 30, 2018; Accepted April 28, 2020

DOI: $10.3892 / \mathrm{mmr} .2020 .11287$

\begin{abstract}
A significant decrease in the expression of spinal microRNA-29c (miR-29c), which is responsible for the regulation of oxytocin receptor (OXTR) expression, was observed in nerve injury pain during childbirth. The present study investigates whether spinal miR-29c could be a potential target for the treatment of pain, via the oxytocin (OT)- $\gamma$-aminobutyric acid (GABA) pathway. A spared nerve injury (SNI) rat model was established to induce neuropathic pain, simulating hyperalgesia. Spinal neurons were treated with OT to mimic the hormonal changes in the central nervous system after delivery. A change in the neuronal miniature inhibitory postsynaptic currents (mIPSCs) was observed in neurons, following the silencing of miR-29c or OT treatment with or without OXTR antagonist. The Von-Frey apparatus was used to measure the animal behaviors. Molecular biological experiments and electrophysical recordings in vivo and in vitro were performed to reveal the potential analgesic mechanisms. miR-29c was significantly downregulated (more than 8-fold) in the spinal dorsal horn of delivery+SNI rats compared with the SNI rats. The silencing of miR-29c resulted in increased pain threshold in SNI rats. Bioinformatics analysis indicated that OXTR was a potential target gene of miR-29c. The delivery+SNI rats presented with higher levels of OT in the cerebrospinal fluid compared with SNI rats, which indicated that the OT signaling pathway may participate in pain relief response. The increased expression of OXTR and GABA in delivery+SNI rats were observed in the miR-29c-silenced
\end{abstract}

Correspondence to: $\mathrm{Dr}$ Shiqin $\mathrm{Xu}$ or Dr Xiaofeng Shen, Department of Anesthesiology, Women's Hospital of Nanjing Medical University, Nanjing Maternity and Child Health Care Hospital, 123 Tianfei Alley, Mochou Road, Nanjing, Jiangsu 210004, P.R. China

E-mail: xusqnj@126.com

E-mail: sxf0418@126.com

Key words: microRNA-29c, oxytocin, oxytocin receptor, $\gamma$-aminobutyric acid, pain relief, delivery
SNI rat model, suggesting that the silencing of miR-29c can mediate pain relief by enhancing the OT-GABA pathway. In addition, an electrophysiology assay was performed to assess the mIPSCs in neurons. The silencing of miR-29c in neurons increased the frequency and amplitude of mIPSCs but there was no influence on the decay time, which suggested that the spinal inhibitory neurons became more active, subsequently reducing the feeling of pain. The inhibition of OXTR reversed the enhanced inhibitory postsynaptic currents, indicating a crucial role for OXTR in the miR-29c-associated pain regulation. Taken together, the results of the present study suggested that spinal oxytocinergic inhibitory control plays an important role in pain relief in the neuropathic pain rat model undergoing vaginal delivery. Silencing spinal miR-29c may be a potential target for pain relief through the OT-GABA pathway.

\section{Introduction}

Chronic post-surgery pain (CPSP) is a common complication that occurs in $\sim 10-50 \%$ of patients after surgery (1). CPSP not only affects the quality of life and work efficiency of patients, but also triggers depression, anxiety and other adverse emotional problems (2). These negative emotions caused by CPSP lead to incidences of suicide as high as $10-15 \%$ (3). CPSP also brings heavy burden to the economy of the medical system and society, it is reported $>€ 200$ billion in Europe and $\$ 150$ billion in America every year (4). The mechanism of CPSP is complex and there is no satisfactory treatment yet. Therefore, it is important to explore new mechanisms and treatment options for pain that can effectively prevent the occurrence of CPSP $(5,6)$.

The natural process of childbirth can lead to serious maternal tissue trauma. Currently approximately one-third of childbirths are achieved through a caesarean section. Approximately $20 \%$ of puerperae have postpartum back pain following caesarean delivery and $\sim 5 \%$ of puerperae have varying degrees of abdominal pain (7). The feelings of pain occur at $\sim 3$ months or 10 months postpartum (19 and $12 \%$, respectively). The aforementioned data reveal that child delivery, including caesarean section is the main factor of chronic pain in puerperae. However, it is noted that the 
incidence of chronic pain caused by childbirth is far less than expected. The incidence of chronic pain after caesarean section was much lower compared with other surgeries, such as transcervical hysterectomy or inguinal hernia repair (8). Thus, a protective mechanism may exist for pain relief during childbirth, which may provide clues for the prevention of CPSP.

A previous study indicated that the protective mechanisms associated with childbirth may be mediated by the analgesic effect of hormones that are synthesized and released in the central nervous system after delivery (9). Tissue injury and inflammation lead to an increased level of cytokines, growth factors or hormones that promote the expression and function of central cannabinoid and opioid receptors, which was considered as a possible mechanism of the analgesic effect $(10,11)$. However, the specific mechanism of postpartum pain relief is unclear. Understanding the mechanism of postpartum pain cannot only provide the molecular basis of the pathophysiological process of CPSP, but also provide a new theoretical basis and therapeutic drug target for the prevention and treatment of CPSP.

In recent years, the discovery of microRNAs (miRNAs/ miRs) provided novel insights for studying disease mechanisms. These non-coding RNAs are involved in the regulation of genome expression by negatively regulating downstream target genes (12). The role of miRNAs in the pathogenesis of pain is largely unknown. The discovery of pain-relief mechanism by miRNAs during childbirth will provide a novel basis for the clinical treatment of pain.

In the present study, microRNAs of spinal cords were screened in rats with neuropathic pain, with delivery and without pregnancy, to identify miRNAs that are associated with delivery-induced pain relief.

\section{Materials and methods}

Animals. Specified pathogen-free Sprague Dawley non-pregnant and pregnant (10-12 days old) female rats were obtained from Vital River Laboratory Animal Technology Co., Ltd (Beijing). Production license no. SCXK (Beijing) 2012-0001; certificate no. 11400700185355. All animal experiments in this study were approved by JiangSu Center for Safety Evaluation of Drug (approval no. LL-20160915-01) in accordance to the 'Regulations for the administration of affairs concerning experimental animals of Jiangsu Province'. The rats were maintained prior to experiments in an animal room under standard conditions $\left(23 \pm 2^{\circ} \mathrm{C}\right.$ temperature, $60 \pm 10 \%$ humidity, $12 \mathrm{~h}$ : $12 \mathrm{~h}$ light-dark cycle). All efforts were made to minimize the suffering of animals. The endpoints for SNI-modelled animals were defined by rapid weight loss, constant unusual response to pain and 24-36 none or less food and water uptake.

Animals were allocated into three groups. Control, non-pregnant rats, $n=12$; SNI, non-pregnant rats, $n=14$; delivery+SNI, pregnant rats, $n=20$. For delivery+SNI group, 32 pregnant rats were SNI-modelled to ensure that at least 20 mice could deliver at day 6 after modelling. The SNI modelling and sham operation (for control rats) were performed at day 0 . The paw withdrawal threshold (PWT) was measured before, immediately after and every two days after modelling, until day 20. The delivery occurred at day 6 after modelling and samples for biological assays were collected at day 9 .

Primary spinal cord dorsal horn neuron isolation and culture. Fetal rats were anesthetized with isoflurane (3\% for induction and 5\% for deep anesthesia) and subsequently decapitated. The back of the rat was sterilized with ethanol, opened with sterilized surgical instruments and the spinal cord was exposed. Then the spinal cord was removed and digested with trypsin containing $0.25 \%$ EDTA at $37^{\circ} \mathrm{C}$ for $10 \mathrm{~min}$, whilst constantly shaking. The trypsinization was stopped by adding culture media, and cells were centrifuged at $200 \mathrm{x} \mathrm{g}$ at room temperature for $6 \mathrm{~min}$. Spinal neurons were then disseminated into single cells and inoculated on a 24-well plate and cultured in an incubator at $37^{\circ} \mathrm{C}$, under $5 \% \mathrm{CO}_{2}$. The culture medium contained neurobasal + B27 (1\%) (cat. no. A3582801; Gibco; Thermo Fisher Scientific, Inc.), FBS (20\%) (cat. no. 10099141C; Gibco; Thermo Fisher Scientific, Inc.), glutamine (final concentration $2 \mathrm{mmol} / \mathrm{l}$; cat. no. G7513; Sigma-Aldrich; Merck KGaA) and penicillin (100 U/ml)-streptomycin solution $(0.1 \mathrm{mg} / \mathrm{ml})$ (ScienCell Research Laboratories, Inc.; cat. no. 0503). Cultured spinal neurons were starved from $\mathrm{B} 27$ for $6 \mathrm{~h}$ before the addition of $1 \mu \mathrm{M}$ OT (Sigma-Aldrich; Merck KGaA) for $30 \mathrm{~min}(13)$.

SNI model establishment. The SNI rat model was constructed as previously described (14). Briefly, after anaesthetizing the rats (3\% isoflurane for induction and $2 \%$ for anesthesia maintenance), the furs on the left leg were shaved with a razor. The skin of the leg was sterilized by alcohol-soaked cotton, before making an incision on the skin $(15-25 \mathrm{~mm})$ from the proximal femur, followed by blunt dissection of the muscle with a surgical scissor. The peroneal nerve and tibial nerve were isolated and ligated. Nerve tissue $(2 \mathrm{~mm})$ was intercepted. The wounds were sealed and disinfected and rats were returned to the cage. The sham operation was performed on control rats, using the same procedure as with the SNI modelling, however the nerve tissue was not intercepted.

Behavioral testing. The mechanical PWT was examined in all rats every two days after the establishment of SNI model. Briefly, rats were placed in a cylinder. The calf muscle of the sural nerve region was vertically penetrated with a VonFreys touch wire (Dynamic). The pain threshold was the intensity of the touch wire specifications when the rats withdrew their legs.

RNA extraction. Treated primary neurons or spinal cord tissues were washed with PBS three times. Subsequently, $1 \mathrm{ml}$ TRIzol (Takara Bio, Inc.) was added, and incubated for $2 \mathrm{~min}$ at RT. The solution was then transferred to an RNase-free Eppendorf tube and $200 \mu \mathrm{l}$ chloroform was added, followed by vigorous shaking for $15 \mathrm{sec}$. Samples were then centrifuged at $12,000 \mathrm{x} \mathrm{g}$ and $4^{\circ} \mathrm{C}$ for $15 \mathrm{~min}$. After centrifugation, the solution was divided into three layers and the upper colorless liquid was carefully transferred into a new Eppendorf tube. An equal volume of isopropanol was added and mixed at room temperature for $5 \mathrm{~min}$, followed by centrifuged at $4^{\circ} \mathrm{C}, 12,000 \mathrm{x} \mathrm{g}$, for $10 \mathrm{~min}$. Following removal of the supernatant, $75 \%$ ethanol $(1 \mathrm{ml})$ was added to the precipitate, and agitated for $15 \mathrm{sec}$ and centrifuged at $12,000 \mathrm{xg}$ and $4^{\circ} \mathrm{C}$. Precipitation was transferred 
into a clean hood and dried for 3-5 min. Thereafter, RNAs were dissolved in DEPC water (Thermo Fisher Scientific, Inc.) and Nanodrop test (Thermo Fisher Scientific, Inc.) was used to determine the RNA concentration.

miRNA microarray analysis. To prepare for the miRNA microarray analysis, the reaction was prepared using miRNA complete Labeling and Hyb kit (24X; Agilent Technologies, Inc.; cat. no. 5190-0456) through dephosphorylation, denaturation and ligation, according to Agilent miRNA microarray protocol. Subsequently, the labelled RNA was purified using Micro Bio-Spin 6 (Bio-Rad Laboratories, Inc.; cat. no. 732-6221) and dried using a vacuum concentrator at $45-55^{\circ} \mathrm{C}$ or on the medium-high heat setting. After preparing the hybridization samples, hybridization was conducted in the Agilent G2545A Hybridization Oven (Agilent Technologies, Inc.) at $55^{\circ} \mathrm{C}$ for $20 \mathrm{~h}$. In addition, the microarray slides were washed, scanned and finally the images were added to be extracted using the Agilent Feature Extraction software v10.5.1.1 (Agilent Technologies, Inc.) to analyze and generate the miRNAs. In order to perform the differential expression analysis, a DEGseq package tool (15) was applied and the significantly differential miRNAs were identified according to the P-value $(<0.05)$.

Bioinformatic databases. Target genes were predicted with the help of bioinformatics (Target Scan release 7.2: http://www. targetscan.org/vert_72/, PicTar release 2007: https://pictar. mdc-berlin.de/, miRanda release 2010: http://www.microrna. org/microrna/home.do and miRase release 22.1: http://www. mirbase.org/) analysis and combined with the literature.

Lentiviral vector construction and animal injection. For the small interfering (si)RNA experiment, the miR-124a and miR-29c siRNA lentivirus (RNAi-LV) were designed and synthesized with the following sequences: miR-124a, 5'-CUCUGCGUG UUCACAGCGGACCUUGAUUUAAUGUCUAUACAAUUA AGGCACGCGGUGAAUGCCAAGAG-3'; and miR-29c, 5'-ATCTCTTACACAGGCTGACCGATTTCTCCTGGTGTT CAGAGTCTGTTTTTGTCTAGCACCATTTGAAATCGG TTATGATGTAGGGGGA-3'. U6-vshRNA-UBI-GFP was the backbone of the lentiviral vector and the same vector but carrying scrambled sequence (5'-GCACGCGTATACTTCGAT TATCACGTATTCTTGTACGTTCTGTGTTGTCCAGGTGA AGGTTAACTCGTGGATACGACCGTATTGATTG-3') was used as negative control lentivirus (NC-LV). All the lentiviruses were produced by Shanghai GeneChem Co., Ltd. The lentiviral vectors were injected into the rats' spinal cord to create local response 4 days after SNI modelling.

Detecting OT concentration by enzyme-linked immunosorbent assay (ELISA). Cerebrospinal fluid (CSF) was isolated from rats in all groups. Briefly, following anesthesia by $2 \%$ pentobarbital sodium $(80 \mathrm{mg} / \mathrm{kg})$, fur on the head and neck of the rat was shaved and skin was sterilized. Subsequently, the rat's head was fixed on the orientator. A longitudinal incision $(\sim 2 \mathrm{~cm})$ was generated by a scalpel and the dorsal muscles of the neck were separated with scissors. In order to avoid bleeding, the deepest muscles attached to the bone were scraped with the back of the scalpel in order to expose the foramen magnum of the occipital bone. The CSF was extracted directly from the foramen magnum. Then the muscles and skin were sealed and the sulfonamide powder was sprayed at the wound to prevent infection. After the CSF extraction, the same amount of sterilized saline was injected to maintain the original pressure in the spinal cord cavity. The OT concentration in the fluid was detected by ELISA kit (cat. no. ab133050; Abcam), according to the manufacturer's instructions.

Western blotting. Spinal neurons were lysed using RIPA lysis buffer with $1 \%$ PMSF on ice for $30 \mathrm{~min}$ and the lysates were centrifuged at $12,000 \mathrm{x} \mathrm{g}$ and $4^{\circ} \mathrm{C}$ for $5 \mathrm{~min}$ to remove cell debris. The protein concentration was determined by BCA assay. Subsequently, the lysate was heated at $95^{\circ} \mathrm{C}$ for $10 \mathrm{~min}$ with 1X Laemmli sample buffer (Bio-Rad Laboratories, Inc.) and then loaded $30 \mu \mathrm{g}$ on a $10 \%$ gel (Bio-Rad Laboratories, Inc.) for electrophoresis by SDS-PAGE. The proteins were subsequently transferred onto a nitrocellulose membrane (Bio-Rad Laboratories, Inc.) and blocked with 5\% BSA TBST for $1 \mathrm{~h}$ at room temperature. After blocking, the membrane was probed with 1:1,000 primary antibodies for $2 \mathrm{~h}$ at room temperature: OXTR (cat. no. ab87312), GABA (cat. no. ab17413), GABA $_{\mathrm{A}}$ (cat. no. ab33299), c-Fos (cat. no. ab208942) and $\beta$-actin (cat. no. ab8226; all from Abcam) and corresponding horseradish peroxidase-conjugated 1:2,000 goat anti-rabbit or goat anti-mouse secondary antibodies (cat. no. ab97080; cat. no. ab97040 Abcam) at room temperature for 2 h. Protein bands were detected by the Western Lightning ${ }^{\circledR}$ ECL Pro Enhanced Chemiluminescence Substrate (PerkinElmer, Inc.) and imaged using the Tanon 1600/1600R Gel Imaging System (UVP, LLC). The ImageJ (v1.8.0_112; National Institutes of Health) was used to quantify the bands.

Paraffin sectioning and immunohistochemistry. Standard immunohistochemistry was used for c-Fos detection. Fresh spinal cord tissues were collected from rats in all groups. Briefly, rats were anesthetized by $3 \%$ pentobarbital sodium, shaved and sterilized. The rats were euthanized by $\mathrm{CO}_{2}(0.5 \mathrm{l} / \mathrm{min}$ in a $5-1$ chamber $)$ inhalation and $2 \mathrm{~cm}$ of $\mathrm{T}_{5}-\mathrm{L}_{5}$ spinal cords were collected. The peripheral soft tissue was removed immediately and cleaned spinal cord tissue was dehydrated using a graded series of ethanol $(50,70$, 85,90 and $100 \%$ ), and then paraffin-embedded and cut into $300-\mu \mathrm{m}$ sections. Heat-mediated antigen retrieval for $20 \mathrm{~min}$ at $100^{\circ} \mathrm{C}$ was achieved using citrate buffer solution $(\mathrm{pH} 6.0)$ (Wuhan Servicebio Technology Co., Ltd.) for c-Fos detection. Sections were incubated with $3 \% \mathrm{H}_{2} \mathrm{O}_{2}$ at room temperature (Wuhan Servicebio Technology Co., Ltd.) for 25 min to block endogenous peroxidase activity. After blocking for $30 \mathrm{~min}$ with 3\% BSA (Beijing Solarbio Science \& Technology Co., Ltd.), paraffin sections were incubated overnight at $4^{\circ} \mathrm{C}$ with 1:200 c-Fos primary antibody (cat. no. ab208942; Abcam). The next day, sections were washed with PBS and subsequently incubated with corresponding HRP-labelled 1:2,000 secondary antibody (Bio-Rad Laboratories, Inc.) for $50 \mathrm{~min}$ at room temperature. The target antigen was detected by 3,3'-diaminobenzidine (DAB) (Agilent Technologies, Inc.) and the nucleus was stained at room temperature by Harris hematoxylin (Wuhan Servicebio Technology Co., Ltd.) for $3 \mathrm{~min}$. 
Table I. Primer sequences.

\begin{tabular}{lll}
\hline Gene & \multicolumn{1}{c}{ Forward sequence (5' to 3') } & \multicolumn{1}{c}{ Reverse sequence (5' to 3') } \\
\hline$G A B A$ & TGTAAACTCAGATGTCCCGCAG' & CGGGCTTGTCCAGCAGAAATA \\
$O X T R$ & CGTACTGGCCTTCATCGTGT & GAATTCTCCTCTCCGCCCAC \\
$G A B A_{A}$ & AGACTCAGGATGGGCCTGAT & GCCCTTCTCCTCCAGTTCAC \\
$c-F o s$ & TTATTTTGGCAGCCCACCGA & TCAAGTCCAGGGAGGTCACA \\
Actin & CCTGGCACCCAGCACAAT & GCCGATCCACACGGAGTACT
\end{tabular}

OXTR, oxytocin receptor; GABA, $\gamma$-aminobutyric acid.

Luciferase assay. The Oxtr minimal promoter or $\mathrm{CpG}$ mutated (C to A) Oxtr minimal promoter was cloned into pGL3-Basic Vector (Promega Corporation) between Xba I and HindIII sites, according to manufacturer's protocol. The fused vector was transfected into $E$. coli by the electrical shock method for amplification. The amplified vector was subsequently collected by GeneJet Plasmid Miniprep kit (Thermo Fisher Scientific, Inc.), according to manufacturer's protocol.Primary neurons were transfected with $1 \mu \mathrm{g}$ prepared luciferase vector and $4 \mu 1$ FuGENE (Promega Corporation) according to manufacturer's protocol. Thereafter, cells were infected with or without miR-29c lentivirus. Cells were lysed by lysis buffer (Promega Corporation) and Renilla luciferase activity was measured after $24 \mathrm{~h}$ using Lucetta Luminometer (Lonza AAL-1001).

Enhanced green fluorescent protein (EGFP) observation in spinal cord tissue. Rats with SNI rats were sacrificed by $\mathrm{CO}_{2}$ inhalation $(0.5 \mathrm{l} / \mathrm{min}$ in a $5-1$ chamber) one week after lentivirus injection. The spinal cord was collected as aforementioned. The tissue was subsequently placed under fluorescence microscopy (magnification, x100) to capture EGFP images.

Electrophysiology assay. The experiment was recorded under whole cell patch clamp mode (Molecular Devices, LLC) in which stereomicroscope (magnification, x10) was used. The tip of the microelectrode, with a diameter of $1.5 \mathrm{~mm}$ and resistance of 7-10 megohms, was placed next to the cultured primary spinal cord neurons under the guidance of the resistance change in the Axon pCLAMP 11.1 software (DL Naturegene Life Sciences, Inc.). When encountering cells, the test pulse square wave appears, and the increase in resistance generates negative pressure to form a giant seal (Giga-Seal). After the formation of a giant resistance seal, a short negative pressure is applied to the micro-electrode chamber, the cells are aspirated and the whole-cell pattern is formed. The membrane current was amplified by an amplifier (Molecular Devices, LLC) and converted to a digital signal by a digitizer (Molecular Devices, LLC). Data were recorded and analyzed using Molecular Devices' Pclamp 10.2 software.

Quantitative (q)PCR. Total RNA was extracted from treated primary neurons or spinal cord tissues as above. qPCR was performed using SYBR ${ }^{\circledR}$ Green Real-time PCR Master mix (Takara) in the StepOnePlus Real-Time PCR system (Applied
Biosystems, Inc.). The quantitative PCR conditions were as follows: $95^{\circ} \mathrm{C}$ for $10 \mathrm{~min}$, followed by 40 cycles of $15 \mathrm{sec}$ at $95^{\circ} \mathrm{C}$ and $60^{\circ} \mathrm{C}$ for $30 \mathrm{sec}$. Actin housekeeping gene was measured as an internal control for all samples. The relative expression of each microRNA was calculated using the $2^{-\Delta \Delta \mathrm{Cq}}$ method (16). Each experiment was replicated three times. The forward and reverse sequences of primers are shown in Table I.

Statistical analysis. SPSS v20 (IBM Corp.) and GraphPad Prism version 5.0 (GraphPad Software, Inc.) were used for statistical analysis. All values are expressed as mean $\pm \mathrm{SD}$. The unpaired 2-tailed Student's t-test was used. When comparing among two or more groups, one-way analysis of variance or repeated measures ANOVA test, followed by Tukey's test, was performed. $\mathrm{P}<0.05$ was considered to indicate a statistically significant difference.

\section{Results}

Delivery-induced pain relief in rats with SNI. To confirm if delivery can induce pain relief in animal models, an SNI rat model was established and the mechanical PWT was investigated. C-Fos is a well-documented marker for neural pain in previous literature, as such, c-Fos was used as an indicator of neural pain level in the present study (17). The results showed that rats presented with decreased mechanical PWT after SNI modelling, compared with those in the control group (Fig. 1A). Delivery+SNI rats presented with significantly increased PWT after the delivery of pups (Fig. 1A), while the PWT in SNI group remained very low. However, the PWT of delivery+SNI rats could not recover back to the level of the control group. In addition, the downregulation of c-Fos after delivery was observed in delivery+SNI group compared with the SNI only counterparts by qPCR, which was in corroboration with the trend observed with the PWT (Fig. 1C). These results were further confirmed by western blotting and immunohistochemistry staining (Fig. 1B and D).

miR-29c is associated with pain relief. The spinal cords of SNI rats with or without delivery were collected at the third day after delivery and analyzed using miRNA microarray. The samples were collected at the third day after delivery to minimize any other unknown factors caused by delivery on the delivery day. As shown in Fig. 1A, the pain threshold still remains at an improved level at the third day after delivery, and was significantly higher, compared with the SNI group; thus, 


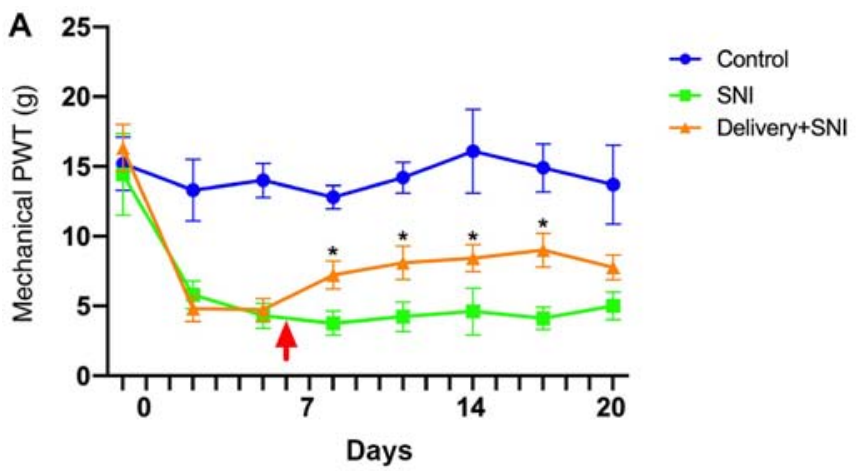

B
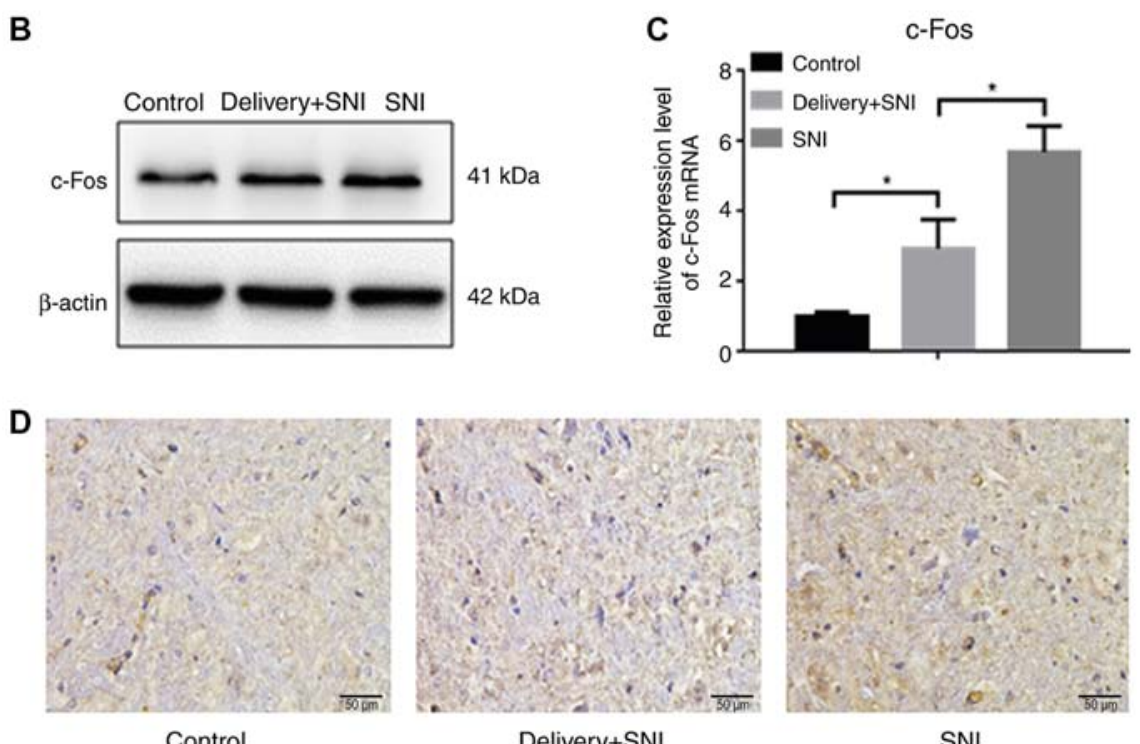

Figure 1. Delivery induces pain relief in SNI model rats. (A) The mechanical PWT in rats was measured before modelling (day 0) and the indicated time points after modelling. Delivery occurred at day 6 after modelling in delivery+SNI group (red arrow). The statistical significance was determined by repeated measures ANOVA test, followed by Tukey's test. Comparisons between the 6-day delivery point and other time points, before and after delivery, were analyzed within each experimental group. The spinal dorsal horn was isolated from 4 rats in each group at day 9 after modelling ( 3 days after delivery) and c-Fos protein level was detected by (B) western blotting, (C) reverse transcription-quantitative PCR and (D) IHC. The IHC images were captured at x200 magnification. Data shown are means of triplicates \pm SD. The statistical significance was determined by one-way ANOVA and Student's t-test. ${ }^{*} \mathrm{P}<0.05$. SNI, spared nerve injury; PWT, paw withdrawal threshold; ANOVA, analysis of variance; IHC, immunohistochemistry.

this time point was considered optimal for collecting samples. The result showed that 71 miRNAs had significant differential expressions between delivery+SNI and SNI groups of rats. There were 37 upregulated miRNAs and 34 downregulated miRNAs in delivery+SNI group (Fig. 2A). qPCR was used to verify 10 miRNAs with more than 5 -fold difference, and the results were consistent with the microarray results (Fig. 2B). The upregulated miR-124a and downregulated miR-29c, which have the largest differences between the two groups, were selected for further investigation. miR-124a-overexpressed and miR-29c-silenced lentiviruses were constructed and injected into SNI rats. The transfection was confirmed by qPCR (Fig. S1). It was found that miR-124a-overexpression had no effects on the pain threshold, but miR-29c-silencing increased the threshold of mechanical PWT $24 \mathrm{~h}$ following transfection (Fig. 2C and D).

miR-29c inhibited OXTR expression. A search online on miRase was performed and it was found that miR-29c is highly conserved during evolution. The base sequence of UAGCACCAUUGAAAUCAGU is highly conserved among humans, rats, mice, dogs and other species (Fig. 3A). The expression levels of miR-29c in different organs were examined and it was found that it is highly expressed in spinal cord, suggesting miR-29c plays an important role in neural tissues (Fig. 3B). Target Scan (http://www.targetscan.org/) analysis revealed a fully matched base sequence 'UGGUGCU' of numerous target genes conserved with the sequence 'ACCACGA' of miR-29c, including spinal oxytocin receptor (OXTR) that is involved in delivery (Fig. 3C). The luciferase assay showed that miR-29c can inhibit the activity of OXTR 3'UTR, suggesting that OXTR might be a target of miR-29c (Fig. 3D).

For further confirmation, the negative control lentivirus, containing a scrambled sequence, and miR-29c-silencing lentivirus was injected into two groups of SNI model rats. miR-29c-silencing lentivirus was detected in spinal cords by green immunofluorescence (Fig. 3E). OXTR expression level in the spinal cord of these rats was analyzed by western blotting. The result showed that rats injected with miR-29c-silencing lentivirus presented with significantly higher OXTR expression compared with SNI rats injected with negative control 

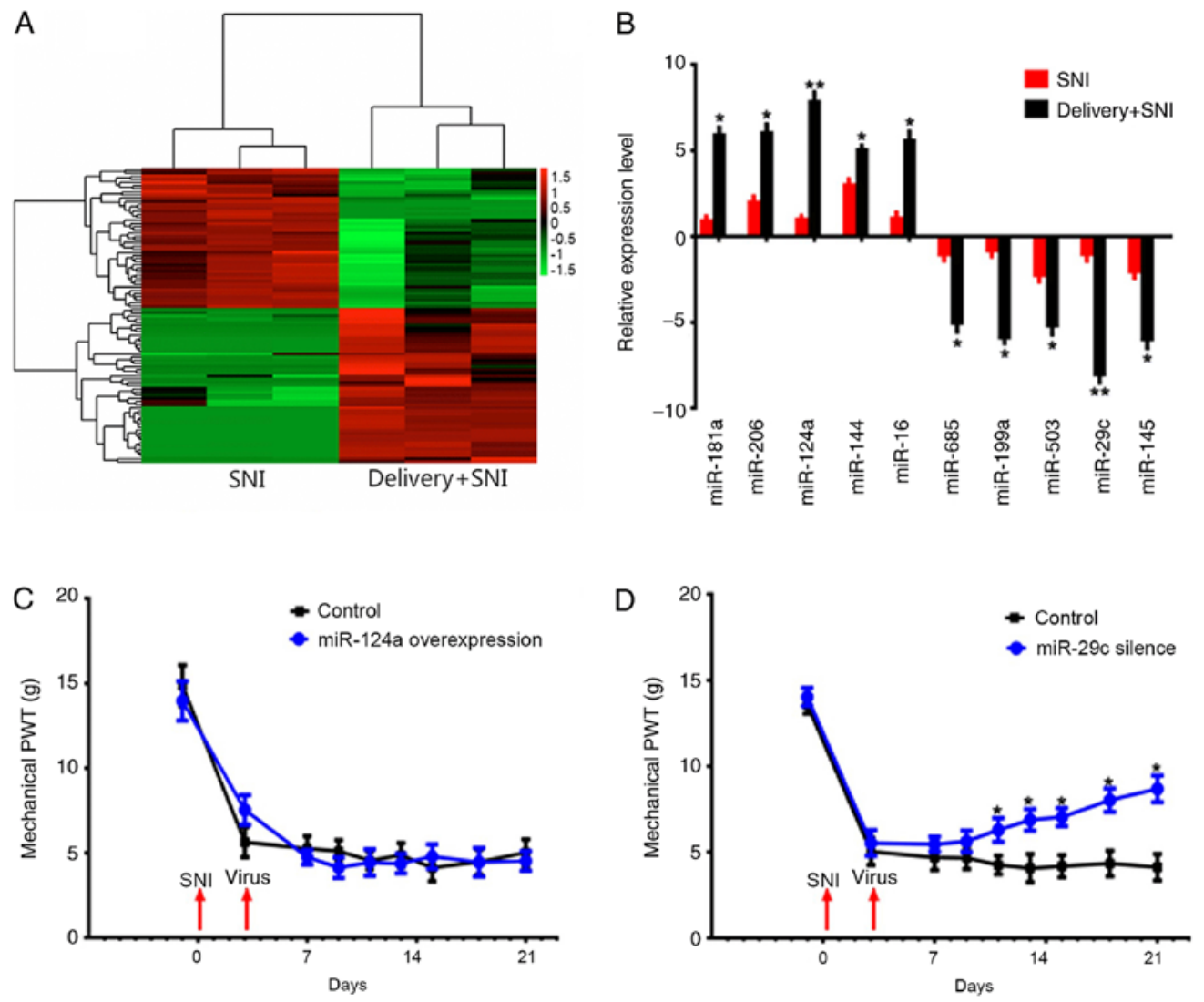

Figure 2. Spinal cord miR-29c is associated with pain relief. (A) The spinal cords of rats from SNI and delivery+SNI groups were extracted 3 days after delivery. The expression of miRNAs in the spinal cord were compared using miRNA chip. Red represents upregulation, while green represents downregulation of miRNA. (B) A total of 10 miRNAs that present more than 5 -fold difference of expression in miRNA chip were selected and verified by qPCR. * $\mathrm{P}<0.05$ and ${ }^{* *} \mathrm{P}<0.01$ vs. SNI group. (C and D) The SNI modelling was performed at day 0 and the miR-124a-overexpression and miR-29c-silencing lentiviruses were constructed and injected into SNI rats at day 4 . The mechanical PWT was tested and the transfection efficiency of the virus was successfully assessed by qPCR at day 11 (1 week following virus injection). ${ }^{*} \mathrm{P}<0.05$. miR/miRNA, microRNA; SNI, spared nerve injury; qPCR, quantitative PCR; PWT, paw withdrawal threshold.

vector lentivirus (Fig. 3F and $\mathrm{G}$ ), confirming that silencing miR-29c can restore OXTR expression in SNI model rats.

Delivery induced OT-GABA mediated pain relief response in neuronal cells. Furthermore, the mechanism of pain relief induced by delivery was investigated. OT has received much attention in recent years, due to its role in spinal antinociception to reduce the symptoms of pain in inflammatory and neuropathic conditions. Therefore, the concentration of OT in CSF was tested, and as expected it was found that on the day of delivery, delivery+SNI rats presented with higher levels of OT in their CSF compared with SNI-only rats (Fig. 4A). This indicated that the OT-GABA pathway may participate in the pain relief response. To mimic the hormone environment of delivery in vitro, primary neuronal cells were isolated from spinal cord of the fetus at day 22 of gestation and treated with OT. The proposed OT-mediated pain relief response was analyzed by measuring the relative protein expression levels and electrophysiology. The results of western blotting and qPCR showed that OT treatment induced the upregulation of GABA, GABA ${ }_{A}$ and OXTR, but the downregulation of c-Fos, indicating the activation of OXTR-mediated pain relief response (Fig. 4B, C and D). Electrophysiology assay was performed to test the miniature inhibitory postsynaptic currents (mIPSCs) in neurons. OT increased the frequency and amplitude of the mIPSCs, however no influences on the decay time were observed (Fig. 4E), which suggested that the spinal inhibitory neurons became more active, subsequently reducing the feeling of pain.

miR-29c reduces pain through the spinal OT-GABA pathway. Based on the aforementioned results, it was hypothesized that the silencing of miR-29c in the spinal cord can alleviate pain, through the synaptic release of GABA, regulating the expression of OXTR. To verify this hypothesis, the expression levels of OXTR, GABA and $\mathrm{GABA}_{\mathrm{A}}$ in the spinal dorsal horn were investigated by western blotting. The results showed a significant increase in the aforementioned activated factors (OXTR and GABA) that are associated with the OT-GABA pathway in SNI rats with spinal injection of miR-29c-silenced lentivirus (Fig. 5A and B). Furthermore, the miR-29c-silencing lentiviral vector and OXTR antagonist were used to intervene cultured neurons, that were treated with OT $(1 \mu \mathrm{M})$ for $30 \mathrm{~min}$. Electrophysiology assay was performed to test mIPSCs in neurons. It was found that silencing miR-29c significantly increased the release of GABA in the culture medium (Fig. 5C) and increased the frequency and amplitude of the mIPSCs compared with the control group (Fig. 5D). Interestingly, the addition of OXTR antagonist L-368,899 hydrochloride ( $2 \mathrm{nM}$ ) (APExBIO) reversed the amplified neuronal inhibitory current flows induced by silencing miR-29c. This result indicated that miR-29c might reduce pain through the OT-GABA pathway (Fig. 5E). 


\begin{tabular}{|c|c|c|c|}
\hline Species & Name & Sequence no. & Base sequence \\
\hline Hsa & has-miR-29c-3p & MIMAT0000681 & 54-UAGCACCAUUUGAAAUCGGUUA-75 \\
\hline mo & mo-miR-29c-3p & MIMAT0000803 & 54-UAGCACCAUUUGAAAUCGGUUA-75 \\
\hline $\mathrm{mmu}$ & mmu-miR-29c-3p & MIMAT0000536 & 54-UAGCACCAUUUGAAAUCGGUUA-75 \\
\hline cfa & cfa-miR-29c & MIMAT0006705 & 37-UAGCACCAUUUGAAAUCGGUUA-58 \\
\hline gga & gga-miR-29c-3p & MIMAT0001183 & 55-UAGCACCAUUUGAAAUCGGU-74 \\
\hline bta & bta-miR-29c & MIMAT0003829 & 54-UAGCACCAUUUGAAAUCGGUUA-75 \\
\hline eca & eca-miR-29c & MIMAT0012964 & 54-UAGCACCAUUUGAAAUCGGUUA-75 \\
\hline ptr & ptr-miR-29c & MIMAT0008082 & 54-UAGCACCAUUUGAAAUCGGUUA-75 \\
\hline $\mathrm{xtr}$ & xtr-miR-29c & MIMAT0003676 & 56-UAGCACCAUUUGAAAUCGGU-75 \\
\hline
\end{tabular}

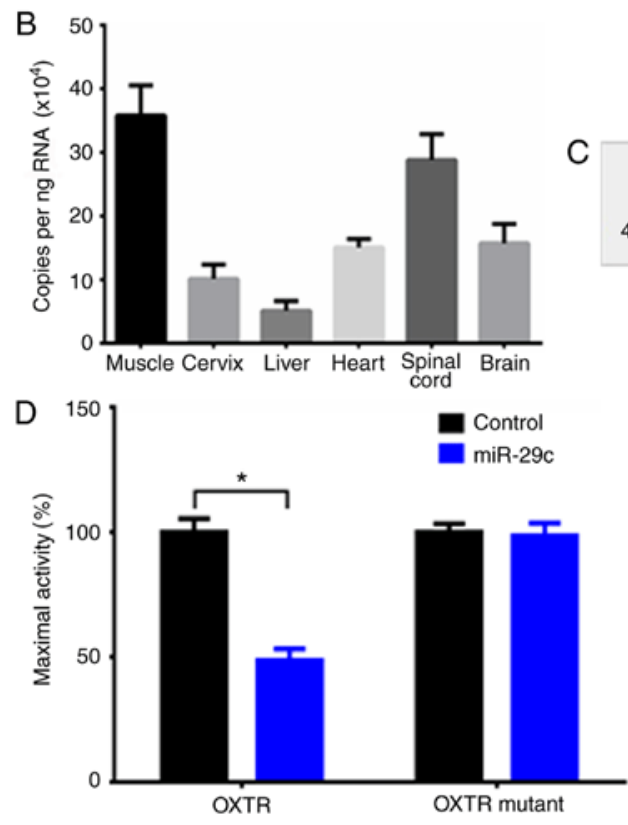

E
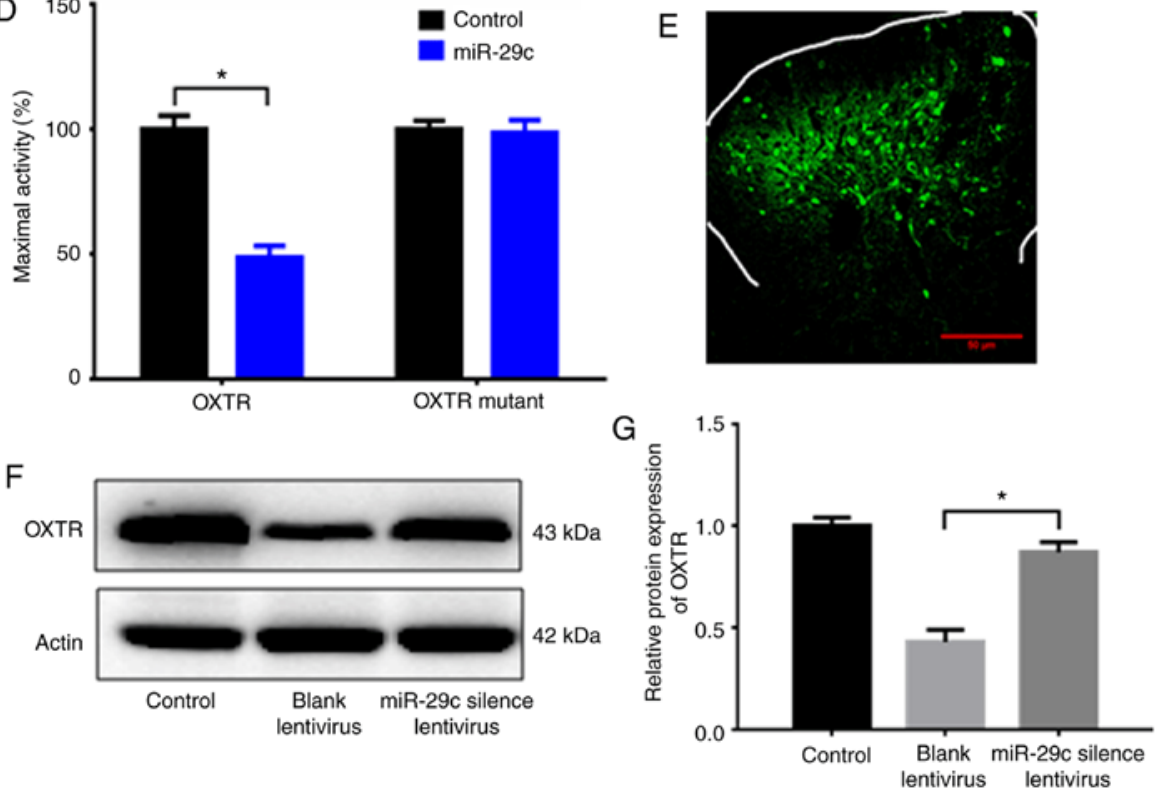

Figure 3. miR-29c regulates OXTR expression. (A) Online analysis (miRase) revealed that miR-29c is highly conserved among human, rat, mouse, dog and other species. (B) The expression of miR-29c in various organs examined by qPCR. (C) A fully matched base sequence 'UGGUGCU' of OXTR and conserved sequence 'ACCACGA' of miR-29c. (D) Luciferase assay measuring the activity of OXTR. (E) SNI rats injected with miR-29c-silencing lentivirus. One week later, the spinal cord of the rat was isolated and the virus was identified by green fluorescence. (F and G) OXTR expression was examined by western blotting. Control, rats with sham operation (not SNI). NC lentivirus, SNI rats injected with NC lentivirus. Silenced miR-29c, SNI rats injected miR-29c silencing lentivirus. Data shown are means \pm SD. Statistical significance was determined by one-way analysis of variance, followed by Tukey's post hoc test. "P $<0.05$. $\mathrm{n}=5$. miR, microRNA; OXTR, oxytocin receptor; SNI, spared nerve injury; NC, negative control.

\section{Discussion}

It has been reported that the incidence of chronic pain caused by childbirth is far below expected compared with other tissue lesions, however the mechanism remains unclear. This project explored the association between pain relief and childbirth. In recent years, the discovery of miRNAs has provided a new avenue for studying disease mechanism. miRNAs are mainly involved in the regulation of gene expression at the post-transcriptional level through the negative regulation of downstream target genes and play important roles in the field of life sciences. Thus far, studies on miRNAs are mainly involved in tumorigenesis, cell differentiation and cardiovascular complications $(12,18,19)$. Recently, the role of miRNAs in pain regulation has begun to draw attention. For example, there were reports that the upregulation of miR-146 and miR-183 in the central nervous system can reduce the expression of inflammatory mediators, which can effectively alleviate pain caused by osteoarthritis (20); blocking the expression of miRNA-21 could effectively alleviate 

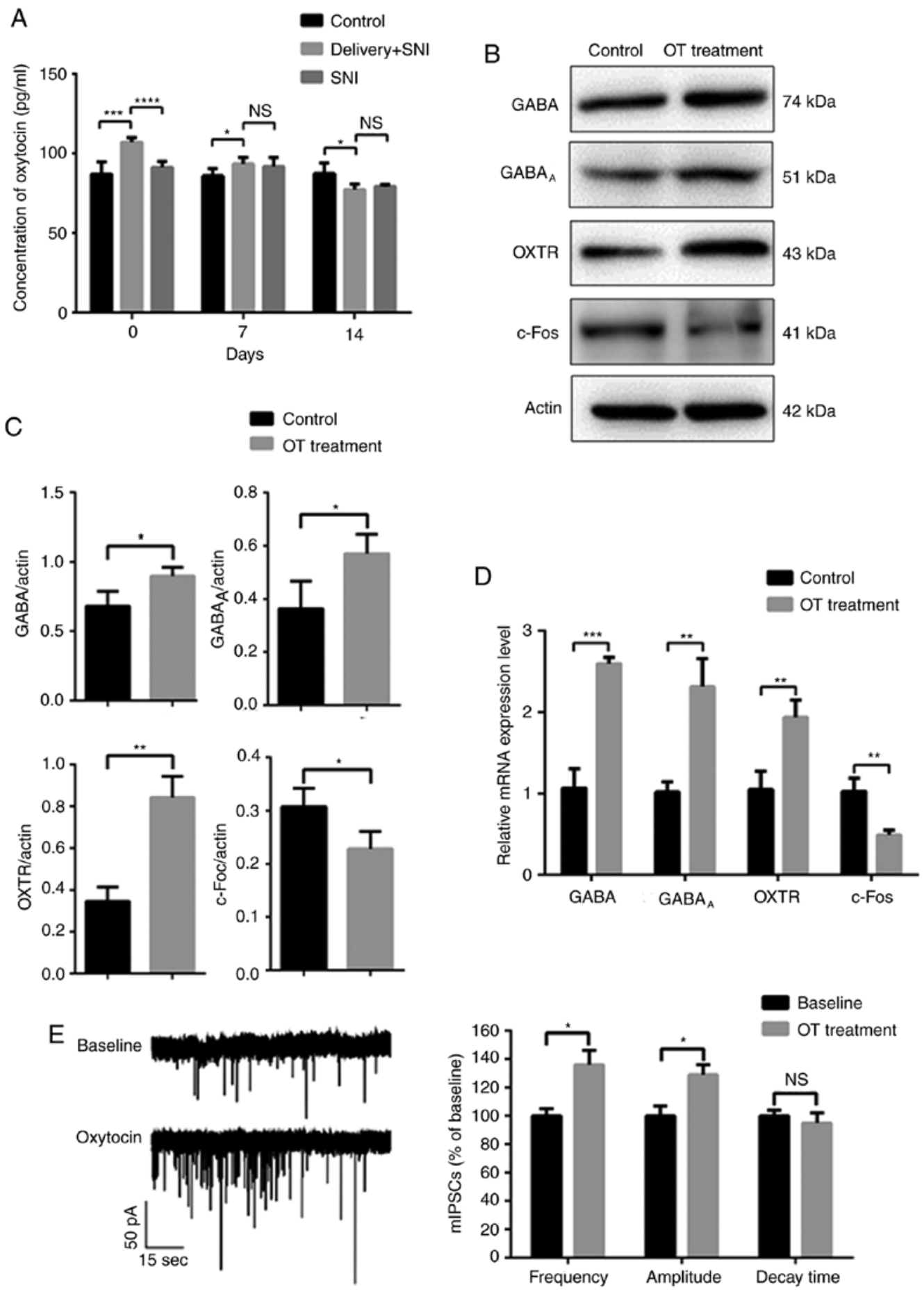

Figure 4. OT induces OXTR-mediated pain relief response in neuronal cells. (A) The cerebrospinal fluid of rats was collected at indicated time points after delivery and OT concentration was examined. (B) Isolated primary neuronal cells were treated with OT and indicated proteins were analyzed by western blotting and (C) the relative protein/actin expression levels were presented. (D) The protein expression levels were also analyzed by reverse transcription-quantitative PCR. (E) mIPSCs in primary neuronal cells analyzed after OT treatment. Data are shown as mean \pm SD. Statistical significance was determined by Student's t-test. NSP $>0.05 ;{ }^{*} \mathrm{P}<0.05 ;{ }^{* *} \mathrm{P} \leq 0.01 ;{ }^{* * *} \mathrm{P} \leq 0.001 ;{ }^{* * * * *} \mathrm{P} \leq 0.0001$. GABA, $\gamma$-aminobutyric acid; OXTR, oxytocin receptor; OT, oxytocin; mIPSCs, miniature inhibitory postsynaptic currents.

neuropathic hyperalgesia (21). In addition, the differentially expressed miRNAs could also be presented and easily detected in the body fluid of patients, in the form of exosomes or cell-free miRNAs, which provides a potentially non-invasive detection method for patients $(22,23)$. In the study, an SNI rat model was constructed during pregnancy to mimic the change in peripheral nerve injury-induced hypersensitivity during the postpartum period. For the control, sham operation was performed on control rats, in an attempt to minimize the differences between control, SNI and delivery+SNI groups. The sham operation may have some impacts on the pain sensitivity of control rats initially but with recovery in the following days, this impact was diminished and the pain threshold of control rats returned to normal. The rats in the 

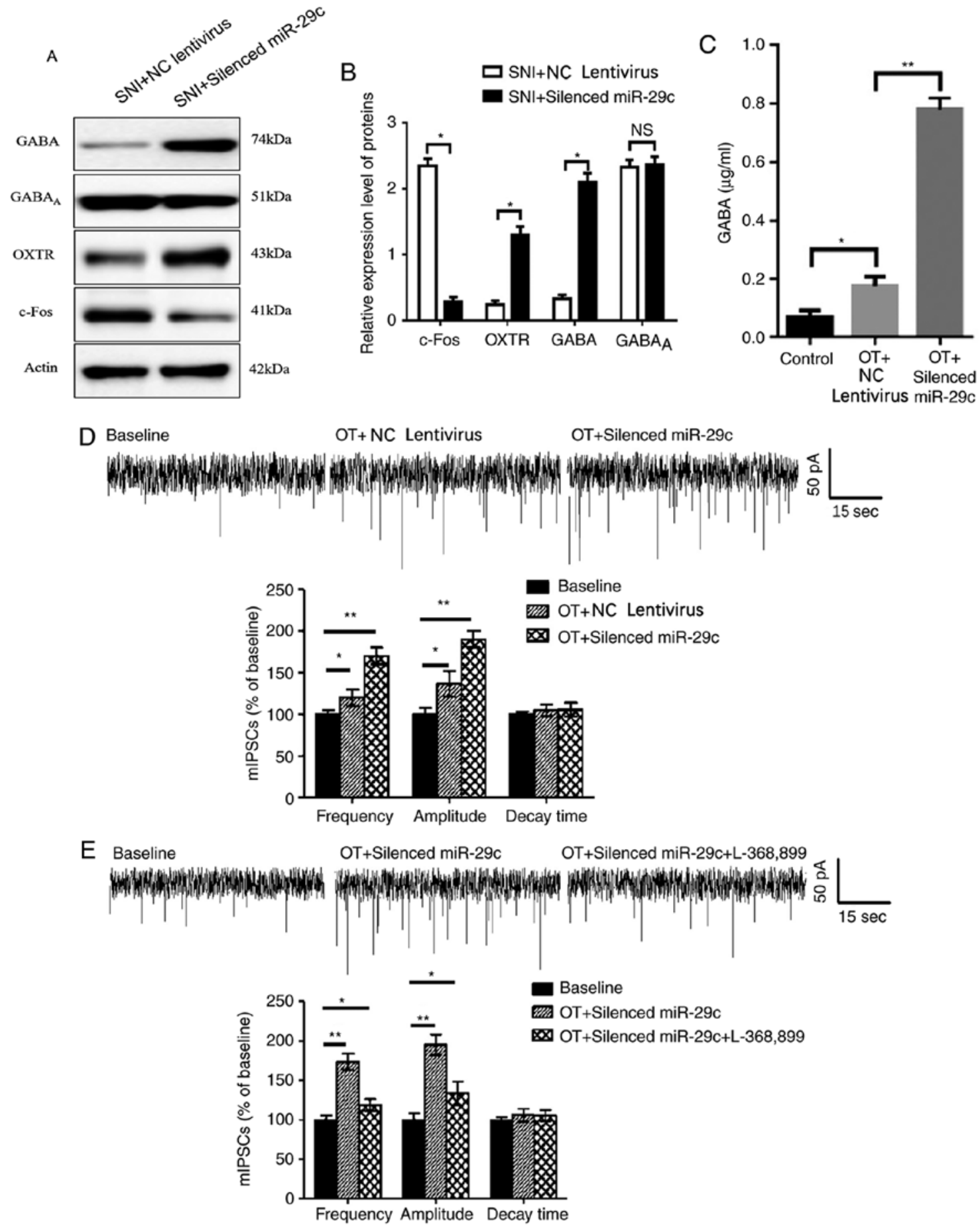

Figure 5. miR-29c regulates pain through spinal oxytocin-GABA pathway. (A and B) SNI rats were injected with miR-29-silencing lentivirus or NC lentivirus. One week later, rats were sacrificed and the expressions of c-Fos, OXTR, GABA and GABAA in spinal dorsal horn were analyzed by western blotting. Data shown are mean \pm SD of triplicates. Statistical significance was determined by Student's t-test. (C) Cultured neurons were pre-treated with OT (1 $\mu \mathrm{M})$ for $30 \mathrm{~min}$ and subsequently intervened by miR-29c-silencing lentiviral or NC lentiviral vectors. The concentration of GABA in the culture medium was measured by enzyme-linked immunosorbent assays. (D) mIPSCs were measured in neurons with same treatments described in (C). (E) Cultured neurons were pre-treated with OT $(1 \mu \mathrm{M})$ for $30 \mathrm{~min}$ and subsequently intervened with miR-29c silencing lentiviral vector with or without OXTR antagonist, L-368,899. The mIPSCs were then measured. ${ }^{\text {NS }} \mathrm{P}>0.05 ;{ }^{*} \mathrm{P}<0.05 ;{ }^{* *} \mathrm{P} \leq 0.01$. miR, microRNA; OXTR, oxytocin receptor; SNI, spared nerve injury; GABA, $\gamma$-aminobutyric acid; OT, oxytocin; mIPSCs, miniature inhibitory postsynaptic currents.

delivery+SNI group were not allowed to perform maternal behaviors, as this may have an impact on the OT level, leading to unknown effects to the pain threshold. The rats did not show obvious negative moods or behaviors as their control or SNI-only counterparts. The results showed a significant improvement in the neuropathic hyperalgesia on day 3 after delivery, which was consistent with a previous report (9). A microarray analysis was performed on the spinal cord of rats with neuropathic pain with or without pregnancy at the third day after delivery. As expected, several miRNAs were found to be differentially expressed indicating the potential effects of miRNAs that are associated with pain regulation. 
To understand the specific candidate miRNAs that are responsible for the protective effects of vaginal delivery, upregulated miR-124a and downregulated miR-29c were selected as the microRNAs of interest, since they demonstrated the largest differences $(\sim 8$-fold $)$ between delivery+SNI and SNI groups respectively (Fig. 2B). The lentivirus was applied in order to increase the expression of spinal miR-124a and decrease the expression of spinal miR-29c. The downregulation of spinal miR-29c significantly improved neuropathic hyperalgesia. Furthermore, bioinformatics analysis and previous studies showed that: i) miR-29c was highly conserved during evolution, with $100 \%$ homology in many species (human, rat, dog, horse.), therefore, functional and mechanistic study in animal models can provide a valuable direction for clinical application; ii) the expression levels of miR-29c in different organs were investigated and it was found that miR-29c is highly expressed in spinal cord, suggesting that miR-29c may play an important role in the central nervous system; iii) many studies on miR-29c demonstrated its involvement in tumorigenesis and immunoregulation $(24,25)$. A recent study demonstrated that the downregulation of miR-29c was involved in the initial pain response, through recapitulating the profile of activated microglia and $\mathrm{TNF} \alpha$ (26). These findings indicate that spinal miR-29c is involved in pain relief induced by childbirth.

Furthermore, the prediction of downstream target genes regulated by miR-29c was conducted using bioinformatics and molecular biology techniques. As a result, the conserved sequence 'ACCACGA' of miR-29c matched with the base sequences 'UGGUGCU' of numerous target genes, including spinal OXTR, which is associated with pain relief after delivery (27). The luciferase assay demonstrated that the of luciferase reporter activity with the OXTR 3'UTR was inhibited by miR-29c, indicating that miR-29c can inhibit OXTR expression in rats. Animal models also indicated a negative association between spinal miR-29c and OXTR mRNA expression in SNI rats injected with miR-29c inhibitory lentivirus, confirming that silencing miR-29c can restore OXTR expression level in neuropathic pain. A previous study suggested that the protective mechanism for the reversal of pain after childbirth may be due to the function of spinal OT and OXTR (27). It was reported that OXTR, a G protein-coupled transmembrane receptor, can induce a series of biological effects when combined with OT (28). Previous studies proposed an antinociceptive role for intrathecal OT $(27,28)$, in which OT was synthesized in the paraventricular nucleus and supraoptic nucleus of the hypothalamus, then entered into the CSF and interacted with increased OXTR in the dorsal horn of the spinal cord. This enhanced the release of inhibitory neurotransmitters GABA, resulting in an increased activity of GABAergic interneurons $(29,30)$. The expression of OXTR, GABA and GABA in the spinal dorsal horn was confirmed with western blotting and qPCR. The data of the present study showed a significant increase in OXTR and GABA, which are associated with the OT-GABA pathway in SNI rats with spinal injection of miR-29c-silencing lentivirus, indicating a potential mechanism for pain modulation by miR-29c.

Increased OT concentration in the CSF was observed in the delivery + SNI rats compared with non-pregnant SNI rats. This was similar to a previous study, which showed increased OT release in the central nervous system after delivery (31). In addition, OT was used in the present study to trigger an enhanced GABAergic inhibitory activity in spinal neurons $(13,32)$. Electrophysiology assay was performed to test mIPSCs in neurons. The in vitro data from the present study revealed that postsynaptic mIPSP frequency and amplitude can be increased by OT treatment in primary spinal cord neuron cells by OXTR, which is consistent with the improved hyperalgesia induced by childbirth in SNI rats (9). Furthermore, the downregulation of miR-29c by miR-29c-silencing lentivirus for $5 \mathrm{~h}$ does not only increase the GABA concentration in culture medium but also increases OT-triggered mIPSP frequency and amplitude, suggesting a GABAergic activation in the central nervous system. Interestingly, this OT-induced GABAergic pathway can be blocked by OXTR antagonist. Furthermore, it was confirmed that the downregulation of miR-29c can reverse hyperalgesia though the OT-GABA pathway. However, it should be noted that the present study only demonstrated that miR-29c can regulate the OT-OXTR pathway, leading to pain relief in an animal model; however, whether and how the OT-OXTR pathway regulates miR-29c was not investigated, which should be further investigated.

Taken together, the present study demonstrated a new mechanism of pain modulation, in which delivery reduced hyperalgesia by downregulating the expression of spinal miR-29c. The spinal OT-GABA pathway plays an important role in reversing childbirth-induced pain. Furthermore, the downregulation of miR-29c in the spinal cord can result in the activation of the OT-GABA pathway. Thus, the present study offers new insights into pain research and therapy.

\section{Acknowledgements}

Not applicable.

\section{Funding}

This work was supported by the National Natural Science Foundation of China (grant nos. 81500944, 81600960 and 81271242), the Talent Fund (grant no. 2015-WSW-059) and Nanjing Municipal Health Bureau general project (grant no. YKK14127).

\section{Availability of data and materials}

The datasets used and/or analyzed during the current study are available from the corresponding author on reasonable request.

\section{Authors' contributions}

$\mathrm{CL}$ and XS conceived and designed the experiments, and drafted the manuscript. XW carried out the molecular genetic studies and participated in the sequence alignment. GZ conducted the immunoassays. YZ and XF participated in the sequence analysis and conducted the statistical analysis. SX conceived the study, participated in its design and coordination, and helped draft the manuscript. All authors read and approved the final manuscript. 


\section{Ethics approval and consent to participate}

This study was approved by JiangSu Center for Safety Evaluation of Drug (Approval ID: LL-20160915-01) in accordance to the 'Regulations for the administration of affairs concerning experimental animals of Jiangsu Province'. All efforts were made to minimize suffering of animals.

\section{Patient consent for publication}

Not applicable.

\section{Competing interests}

The authors declare that they have no competing interests.

\section{References}

1. Rashiq S and Dick BD: Post-surgical pain syndromes: A review for the non-pain specialist. Can J Anaesth 61: 123-130, 2014.

2. Salama-Hanna J and Chen G: Patients with chronic pain. Med Clin North Am 97: 1201-1215, 2013.

3. Rihmer Z: Antidepressants, depression and suicide. Neuropsychopharmacol Hung 15: 157-164, 2013 (In Hungarian).

4. Tracey I and Bushnell MC: How neuroimaging studies have challenged us to rethink: Is chronic pain a disease? J Pain 10: $1113-1120,2009$

5. Marchand F, Jones NG and McMahon SB: Future treatment strategies for neuropathic pain. Handb Exp Pharmacol: 589-615, 2009.

6. Kaasa T, Romundstad L, Roald H, Skolleborg K and Stubhaug A: Hyperesthesia one year after breast augmentation surgery increases the odds for persisting pain at four years A prospective four-year follow-up study. Scand J Pain 1: 75-81, 2010.

7. Hannah ME, Whyte H, Hannah WJ, Hewson S, Amankwah K, Cheng M, Gafni A, Guselle P, Helewa M, Hodnett ED, et al: Maternal outcomes at 2 years after planned cesarean section versus planned vaginal birth for breech presentation at term: The international randomized term breech trial. Am J Obstet Gynecol 191: 917-927, 2004.

8. Eisenach JC, Pan P, Smiley RM, Lavand'homme P, Landau R and Houle TT: Resolution of pain after childbirth. Anesthesiology 118: 143-151, 2013.

9. Gutierrez S, Liu B, Hayashida K, Houle TT and Eisenach JC: Reversal of peripheral nerve injury-induced hypersensitivity in the postpartum period: Role of spinal oxytocin. Anesthesiology 118: $152-159,2013$

10. Lavand'homme P: Chronic pain after childbirth. Curr Opin Anaesthesiol 26: 273-277, 2013.

11. Gintzler AR and Liu NJ: Importance of sex to pain and its amelioration; relevance of spinal estrogens and its membrane receptors. Front Neuroendocrinol 33: 412-424, 2012

12. Chiu H, Alqadah A and Chang C: The role of microRNAs in regulating neuronal connectivity. Front Cell Neurosci 7: 283, 2014.

13. Lin YT, Huang CC and Hsu KS: Oxytocin promotes long-term potentiation by enhancing epidermal growth factor receptor-mediated local translation of protein kinase $\mathrm{M} \zeta$. J Neurosci 32: 15476-15488, 2012.

14. Decosterd I and Woolf CJ: Spared nerve injury: An animal model of persistent peripheral neuropathic pain. Pain 87: 149-158, 2000

15. Wang L, Feng Z, Wang X, Wang X and Zhang X: DEGseq: An $R$ package for identifying differentially expressed genes from RNA-seq data. Bioinformatics 26: 136-138, 2010.
16. Livak KJ and Schmittgen TD: Analysis of relative gene expression data using real-time quantitative PCR and the 2(-Delta Delta C(T)) method. Methods 25: 402-408, 2001.

17. Harris JA: Using c-fos as a neural marker of pain. Brain Res Bull 45: 1-8, 1998 .

18. Braicu C, Cojocneanu-Petric R, Chira S, Truta A, Floares A, Petrut B, Achimas-Cadariu P and Berindan-Neagoe I: Clinical and pathological implications of miRNA in bladder cancer. Int J Nanomedicine 10: 791-800, 2015.

19. Huang J, Lyu H, Wang J and Liu B: MicroRNA regulation and therapeutic targeting of survivin in cancer. Am J Cancer Res 5: 20-31, 2014

20. Li X, Kroin JS, Kc R, Gibson G, Chen D, Corbett GT, Pahan K, Fayyaz S, Kim JS, van Wijnen AJ, et al: Altered spinal microRNA-146a and the microRNA-183 cluster contribute to osteoarthritic pain in knee joints. J Bone Miner Res 28: 2512-2522, 2013.

21. Sakai A and Suzuki H: Nerve injury-induced upregulation of miR-21 in the primary sensory neurons contributes to neuropathic pain in rats. Biochem Biophys Res Commun 435: 176-181, 2013.

22. Petersen B and Kingsley K: Differential expression of miR-21, miR-133 and miR-155 from exosome fractions isolated from oral squamous cell carcinomas in vitro. J Med Discov 1: jmd16010, 2016.

23. Ma C, Nguyen HPT, Luwor RB, Stylli SS, Gogos A, Paradiso L, Kaye AH and Morokoff AP: A comprehensive meta-analysis of circulation miRNAs in glioma as potential diagnostic biomarker. PLoS One 13: e0189452, 2018.

24. Li W, Yi J, Zheng X, Liu S, Fu W, Ren L, Li L, Hoon DSB, Wang J and Du G: miR-29c plays a suppressive role in breast cancer by targeting the TIMP3/STAT1/FOXO1 pathway. Clin Epigenetics 10: 64, 2018.

25. Liston A, Papadopoulou AS, Danso-Abeam D and Dooley J: MicroRNA-29 in the adaptive immune system: Setting the threshold. Cell Mol Life Sci 69: 3533-3541, 2012.

26. Jeong H, Na YJ, Lee K, Kim YH, Lee Y, Kang M, Jiang BC, Yeom YI, Wu LJ, Gao YJ, et al: High-resolution transcriptome analysis reveals neuropathic pain gene-expression signatures in spinal microglia after nerve injury. Pain 157: 964-976, 2016.

27. Juif PE, Breton JD, Rajalu M, Charlet A, Goumon Y and Poisbeau P: Long-lasting spinal oxytocin analgesia is ensured by the stimulation of allopregnanolone synthesis which potentiates GABA(A) receptor-mediated synaptic inhibition. J Neurosci 33: 16617-16626, 2013

28. Gimpl G and Fahrenholz F: The oxytocin receptor system: Structure, function, and regulation. Physiol Rev 81: 629-683, 2001.

29. Sun W, Zhou Q, Ba X, Feng X, Hu X, Cheng X, Liu T, Guo J, Xiao L, Jiang J, et al: Oxytocin relieves neuropathic pain through GABA release and presynaptic TRPV1 inhibition in spinal cord. Front Mol Neurosci 11: 248, 2018.

30. Severino AL, Chen R, Hayashida K, Aschenbrenner CA, Sun H, Peters CM, Gutierrez S, Pan B and Eisenach JC: Plasticity and function of spinal oxytocin and vasopressin signaling during recovery from surgery with nerve injury. Anesthesiology 129: 544-556, 2018

31. Takeda S, Kuwabara Y and Mizuno M: Effects of pregnancy and delivery on oxytocin levels in human plasma and cerebrospinal fluid. Endocrinol Jpn 32: 875-880, 1985.

32. Han RT, Kim YB, Park EH, Kim JY, Ryu C, Kim HY, Lee J, Pahk K, Shanyu C, Kim H, et al: Long-term isolation elicits depression and anxiety-related behaviors by reducing oxytocin-induced GABAergic transmission in central amygdala. Front Mol Neurosci 11: 246, 2018

(i)(5) This work is licensed under a Creative Commons Attribution-NonCommercial-NoDerivatives 4.0 International (CC BY-NC-ND 4.0) License. 